\title{
Intra- and Intermolecular Proton Transfer in 1H(2H)-1,2,3-Triazole Based Systems
}

\author{
Zhen Zhou, ${ }^{\dagger, *}$ Rui Liu, ${ }^{\dagger, \dagger}$ Jenghan Wang, ${ }^{\dagger}$ Siwen Li, ${ }^{\dagger}$ Meilin Liu, ${ }^{*, \dagger}$ and Jean-Luc Brédas ${ }^{*, \hbar}$ \\ School of Material Science \& Engineering, Georgia Institute of Technology, 771 Ferst Drive, NW, \\ Atlanta, Georgia, and School of Chemistry \& Biochemistry, Georgia Institute of Technology, 770 State Street, \\ Atlanta, Georgia
}

Received: December 12, 2005; In Final Form: January 15, 2006

\begin{abstract}
Hartree-Fock ab initio and density functional theory calculations suggest that intra- and intermolecular proton transfer between neutral and protonated triazole tautomers play a vital role in the observed dramatic proton conduction in triazole-based polymer electrolyte membranes.
\end{abstract}

It has recently been reported that $1 H(2 H)$-1,2,3-triazole can effectively promote proton conduction in a polymer electrolyte membrane (PEM). ${ }^{1}$ In a way similar to $\mathrm{H}_{2} \mathrm{O}, \mathrm{H}_{3} \mathrm{PO}_{4}$, or imidazole used in PEMs, $1 H(2 H)$-1,2,3-triazole may conduct proton through either the vehicle mechanism or Grotthuss mechanism. ${ }^{2}$ The latter is especially important to the design of PEMs with triazole groups attached to the backbones. Because of the demonstrated ability of $1 H(2 H)$-1,2,3-triazole to promote proton transport, triazole-containing PEMs hold great promise for a new generation of PEM fuel cells that could operate at high temperatures (above $100{ }^{\circ} \mathrm{C}$ ) with low relative humidity.

The Grotthuss-type proton motion along an imidazole molecular chain ${ }^{3,4}$ is sketched in Scheme 1. Clearly, once a proton is transferred from one imidazole ring to an adjacent one, reorientation (or flipping) of the imidazole moiety becomes necessary for subsequent intermolecular proton transfer to take place in the same direction. It is this reorientation step that was reported as the rate-limiting step for long-range proton conduction along the molecular chain; it might be the major impediment to high proton conductivity in imidazole-based materials as well. ${ }^{5}$ In contrast, triazole-based systems might open the possibility for different mechanisms, as suggested by the existence of several proton-carrying isomers. Neutral triazole has two tautomers, $1 H$-1,2,3-triazole (A) and $2 H$-1,2,3-triazole (B), and so does protonated triazole, 1,3-di $H$-1,2,3-triazolium (a) and 1,2-di $H$-1,2,3-triazolium (b) cations.

To date, both experimental and theoretical studies ${ }^{6-8}$ of $1 H(2 H)$-1,2,3-triazole have been limited primarily to the exploration of the tautomerism between the neutral isomers $\mathbf{A}$ and B. Little is known about the tautomerism of the 1,2-di $H$ - and 1,3-diH-1,2,3-triazolium cations $\mathbf{a}$ and $\mathbf{b}$ or the interactions among these four species. ${ }^{9}$ To gain an understanding of the longrange proton conduction mechanism, it is necessary to determine how the triazolium cations interact with the neutral triazole molecules and how the short-range proton transfer proceeds in complexes involving more than one triazole unit. In this paper, we report our results on quantum-mechanical calculations of

* Corresponding authors. E-mail: M.L., meilin.liu@mse.gatech.edu; J.-L.B., jean-luc.bredas@chemistry.gatech.edu.

† School of Material Science \& Engineering.

$\doteqdot$ School of Chemistry \& Biochemistry.

\section{SCHEME 1}

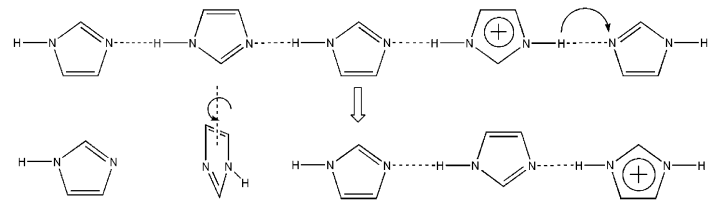

Proton conduction in imidazole through Grotthuss mechanism

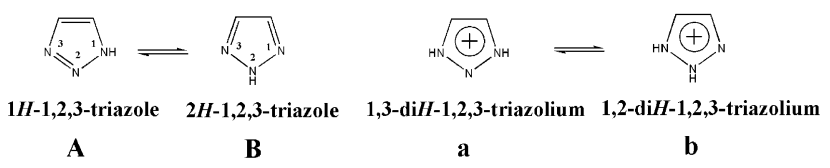

triazole/triazolium dimers, aimed at finding the most probable combinations, transition states of intermolecular proton-transfer process in these dimers, and possible proton-transfer mechanisms responsible for proton conduction in triazole-based systems. The effect of interactions involving trimers and more complex systems will be reported later.

The calculations were carried out with the Gaussian-03 program. ${ }^{10}$ The initial geometries were from the triazole crystal structures determined by X-ray diffraction. ${ }^{11}$ All geometries were fully optimized at the HF/6-31G(d) and B3LYP/6$311+\mathrm{G}(\mathrm{d})$ levels of theory. Harmonic vibrational frequency calculations were then performed at the B3LYP/6-311+G(d) level to confirm the optimized structures as minima and evaluate zero-point energy (ZPE) corrections.

First, we reproduced the results of previous calculations ${ }^{7}$ on the various tautomers $(\mathbf{A}, \mathbf{B}, \mathbf{a}$, and $\mathbf{b})$. For neutral triazole in the gas phase, $\mathbf{B}$ is more stable than $\mathbf{A}$ by $18.6 \mathrm{~kJ} / \mathrm{mol}$. Because A $(\mu=4.64 \mathrm{D})$ has a significantly larger dipole moment than B $(\mu=0.21 \mathrm{D})$, however, A becomes increasingly more stable with the medium polarity. As a protonated cation, tautomer a is more stable than b by $49.1 \mathrm{~kJ} / \mathrm{mol}$. The $\mathbf{A} \Leftrightarrow \mathbf{B}$ or $\mathbf{a} \Leftrightarrow \mathbf{b}$ tautomerisms may greatly assist in the inter- and intramolecular proton transfer in the system. As has been reported earlier, 8,9 these tautomeric processes may involve either solvent molecules or the formation of dimers, trimers, or other complexes to lower the energy barriers and assist proton conduction. 


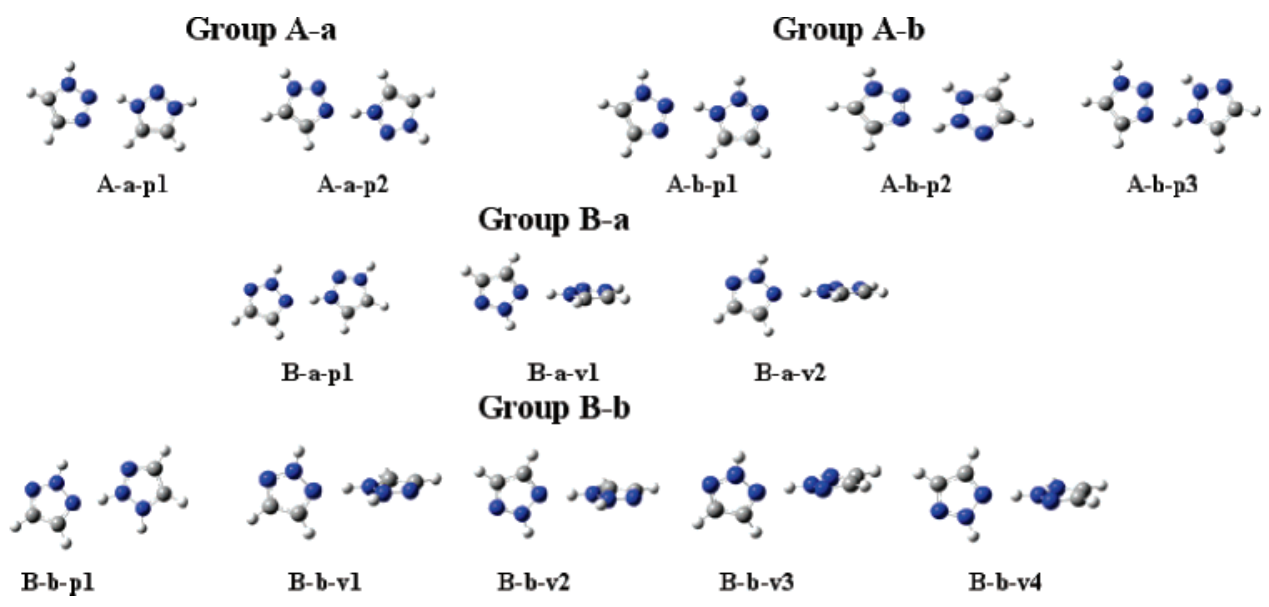

Figure 1. Possible structures of triazole/triazolium dimer complex.

To study the intermolecular proton transfer, initial structures of neutral/protonated triazole complexes were built by combining one of the two neutral tautomers $(\mathbf{A}$ or $\mathbf{B})$ with one of the two cations (a or $\mathbf{b})$ through a single $\mathrm{N}-\mathrm{H}-\mathrm{-}-\mathrm{N}$ hydrogen bond and varying the dihedral angle between the two molecular planes. To select stable geometries, we performed geometry optimizations at the HF/6-31G(d) level, which led to 13 possible configurations of the complexes; further geometry optimizations and frequency calculations at the B3LYP/6-311+G(d) level produced the complex structures shown in Figure 1, with the corresponding energy values summarized in Table 1 (Supporting Information). We also considered coplanar complexes with two hydrogen bonds between the neutral and protonated triazole molecules as the initial structures. After geometry optimization, however, all these structures reduced to those presented in Figure 1.

We find that stable complexes are formed via hydrogen-bond formation between an $-\mathrm{NH}$ group on the protonated triazole as the proton donor and a nitrogen atom on the neutral triazole as the proton acceptor. The distance between two nitrogen atoms in the hydrogen bond is in the range $2.69-2.75 \AA$. The thirteen structures can be distributed into four groups: $\mathbf{A}-\mathbf{a}, \mathbf{A}-\mathbf{b}, \mathbf{B}-\mathbf{a}$, $\mathbf{B}-\mathbf{b}$. In groups $\mathbf{B}-\mathbf{a}$ and $\mathbf{B}-\mathbf{b}$, some complexes have two optical isomers, in which the two planes of the triazole molecules are perpendicular to each other; tautomer $\mathbf{B}$ has more flexibility to form stable complexes with protonated cations because of its symmetry.

Earlier studies on ionic hydrogen bonds in other systems reported bond strengths in the range $20-150 \mathrm{~kJ} / \mathrm{mol} .{ }^{12}$ Evaluations of the standard Gibbs free energy change for the reaction $(\mathbf{A} / \mathbf{B})+(\mathbf{a} / \mathbf{b}) \rightarrow(\mathbf{A} / \mathbf{B})-(\mathbf{a} / \mathbf{b})$ are given in Table 1 (Supporting Information). In addition, the reaction barriers of these intermolecular proton-transfer processes, $\mathrm{C}_{2} \mathrm{~N}_{3} \mathrm{H}_{4}{ }^{+}+\mathrm{C}_{2} \mathrm{~N}_{3} \mathrm{H}_{3} \rightarrow$ $\mathrm{C}_{2} \mathrm{~N}_{3} \mathrm{H}_{3}+\mathrm{C}_{2} \mathrm{~N}_{3} \mathrm{H}_{4}{ }^{+}$, are computed to be $4-8 \mathrm{~kJ} / \mathrm{mol}$ on the basis of the energies predicted at the B3LYP/6-311+G(d) level with ZPE corrections. The results indicate that the protonated triazole cations in triazole-based materials have a great tendency to form various complexes with neutral triazole molecules through ionic hydrogen bonds. As illustrated in the comparative energy diagram of Figure 2, the most stable structure is $\mathbf{A}-\mathbf{a}-$ p2 whereas the least stable ones are $\mathbf{B}-\mathbf{b}-\mathrm{v} 1 / \mathrm{v} 2$; the energy difference between them is about $64.1 \mathrm{~kJ} / \mathrm{mol}$. The comparative energy diagram allows us to discern three classes of systems: all the $\mathbf{B}-\mathbf{b}$ structures have higher energies; nearly all $\boldsymbol{A}-\boldsymbol{b}$ and $\boldsymbol{B}$ - $\boldsymbol{a}$ structures belong to to a medium-energy class whereas $\mathbf{A}-\mathbf{a}-\mathrm{p} 2$ is the only member of the low-energy class. As shown in Table 1, if proton transfers from the cation to the neutral

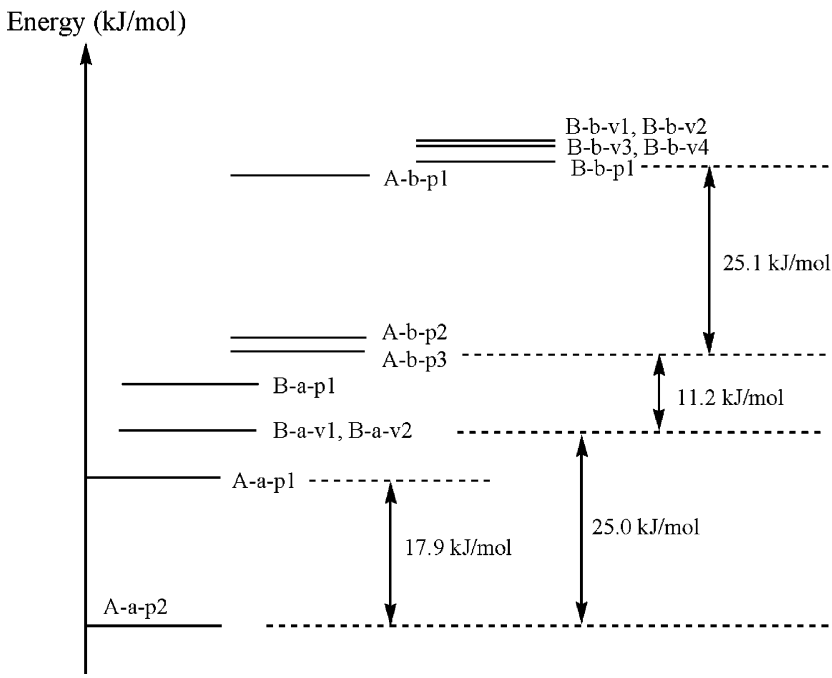

Figure 2. Comparative energy diagram of triazole/triazolium dimer complex.

triazole through the ionic hydrogen bond in the complex, the resulting complex still belongs to the same class. Because the complexes in the same class have very small energy differences $(0-20 \mathrm{~kJ} / \mathrm{mol})$ and the proton-transfer reaction barriers are quite small, we conclude that the intermolecular proton tranfer reactions are feasible for all complexes.

Based on our calculations, $\mathbf{A}-\mathbf{a}-\mathrm{p} 2$ has the lowest energy in the gas phase and is the most likely complex responsible for intermolecular proton transfer under the condition in which tautomer $\mathbf{A}$ is the major species of neutral triazoles, such as in a polar medium. ${ }^{7}$ After a proton transfers from cation a to neutral A through the hydrogen bond, the resulting complex is nearly identical, with $\mathbf{A}$ and a having switched positions. This process is similar to that in an imidazole-containing system.

In a less polar environment, tautomer $\mathbf{B}$ will be more stable than tautomer A. We note that it is possible for the less stable $\mathbf{B}$-b group complexes in the high-energy class to transform to complex $\mathbf{A}-\mathbf{b}-$ p1 through intermolecular proton-transfer reactions. Subsequently, as it is energetically favorable $(\Delta G \approx-23$ $\mathrm{kJ} / \mathrm{mol}), \mathbf{A}-\mathbf{b}-\mathrm{p} 1$ may switch to $\mathbf{A}-\mathbf{b}-\mathrm{p} 3$ via a small rotation of the two triazole molecules and further to $\mathbf{B}-\mathbf{a}-\mathrm{p} 1 / \mathrm{v} 1 / \mathrm{v} 2(\Delta G$ $\approx-11.2 \mathrm{~kJ} / \mathrm{mol}$ ) through another proton-transfer reaction. Thus, complexes such as $\mathbf{B}-\mathbf{a}-\mathrm{p} 1 / \mathrm{v} 1 / \mathrm{v} 2$ could turn out to be the most likely complexes under less polar conditions. However, other complexes (such as $\mathbf{A}-\mathbf{b}-\mathrm{p} 3$ and the $\mathbf{B}-\mathbf{b}$ group complexes) will be involved in the intermolecular proton-transfer process as well. 


\section{SCHEME 2: Proton Transfer in $1 H(2 H)-1,2,3-T r i a z o l e$}
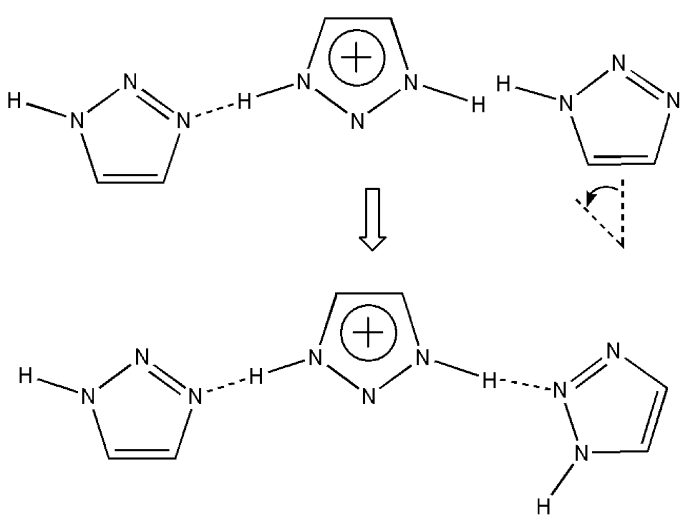

Recently, we have demonstrated ${ }^{1}$ that the proton conductivities of poly(4-vinyl-1H-1,2,3-triazole) are about $10^{5}$ times higher than those of poly(4-vinylimidazole). Based on the present calculations, the intermolecular proton-transfer barriers of both imidazole and triazole are similar and small, about $4-8 \mathrm{~kJ} /$ mol. Therefore, the large difference in proton conductivity between these two materials is unlikely related to the protontransfer barrier, but to other effects. Scheme 2 illustrates that if along the proton-transport direction, atom $\mathrm{N} 1$ on an individual triazole ring is linked to a hydrogen already and cannot accept another arriving proton, the $\mathrm{N} 2$ atom on the ring provides another proton binding site and also has steric flexibility to form intermolecular proton-transfer intermediates. Therefore, compared to imidazole, the reorientation step for the triazole ring is easier. Also, the $\mathbf{A} \Leftrightarrow \mathbf{B}$ and $\mathbf{a} \Leftrightarrow \mathbf{b}$ tautomerisms can occur by interacting with either solvent molecules or neighboring triazole molecules such as from the complex $\mathbf{B}-\mathbf{b}-\mathrm{p} 1$ to $\mathbf{B}-\mathbf{a}-$ p1 or through $\mathbf{A}-\mathbf{A}, \mathbf{B}-\mathbf{B}$, and $\mathbf{A}-\mathbf{B}$ dimers $^{8}$ via double protontransfer reactions. These tautomeric (intramolecular proton transfer) processes, as described in Scheme 2, also result in proton conduction along a certain direction. As a consequence, protons can hop from one triazole ring to another without the need of triazole ring flipping. These proposed proton-transfer mechanisms are plausible but have to be confirmed by more rigorous computation and experimental measurements of the energetics of the processes. Work along these lines is in progress.

In conclusion, we propose that the species responsible for proton conduction in a $1 H(2 H)$-1,2,3-triazole-containing system are the neutral and pronated triazole tautomers as well as various combination of these species. There are many possible pathways and mechanisms available for intra- and intermolecular proton transfers, making triazole a very effective proton conducting group for long-range proton conduction in polymer-electrolyte membranes.
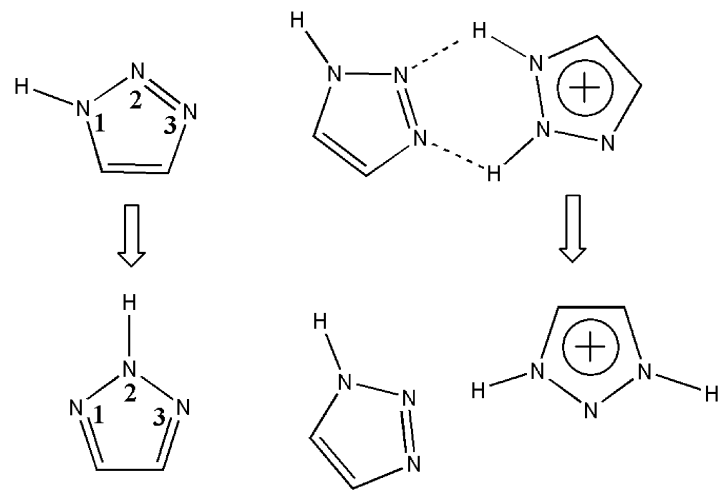

Supporting Information Available: Table 1 of energies calculated at the B3LYP/6-311+G(d) level. This material is available free of charge via the Internet at http://pubs.acs.org.

\section{References and Notes}

(1) Zhou, Z.; Li, S.; Zhang Y.; Liu, M. J. Am. Chem. Soc. 2005, 127, 10824

(2) Kreure, K. D.; Dippel, Th.; Hainovsky, N. G.; Maier, J. Ber Bunsen-Ges. Phys. Chem. 1992, 96, 1736.

(3) Münch, W.; Kreuer, K. D.; Silvestri, W.; Maier, J.; Seifert, G. Solid State Ionics 2001, 145, 437.

(4) Iannuzzi, M.; Parrinello, M. Phys. Rev. Lett. 2004, 93, 025901.

(5) Goward, G. R.; Schuster, M. F. H.; Sebastiani, D.; Schnell, I.; Spiess, H. W. J. Phys. Chem. B 2002, 106, 9322.

(6) Catalán, J.; Sánchez-Cabezudo, M.; De Paz, J. L. G.; Elguero, J.; Taft, R. W.; Aniva, F. J. J. Comput. Chem. 1989, 10, 426.

(7) Abboud, J. M.; Foces-Foces, C.; Notario, R.; Trifonov, R. E.; Volovodenko, A. P.; Ostrovskii, V. A.; Alkorta, I.; Elguero, J. Eur. J. Org. Chem. 2001, 3013.

(8) Rauhut, G. Phys. Chem. Chem. Phys. 2003, 5, 791.

(9) Alkorta, I.; Elguero, J. J. Chem. Soc., Perkin Trans. 2 1998, 2497.

(10) Frisch, M. J.; Trucks, G. W.; Schlegel, H. B.; Scuseria, G. E.; Robb M. A.; Cheeseman, J. R.; Zakrzewski, V. G.; Montgomery, J. A., Jr.; Stratmann, R. E.; Burant, J. C.; Dapprich, S.; Millam, J. M.; Daniels, A. D.; Kudin, K. N.; Strain, M. C.; Farkas, O.; Tomasi, J.; Barone, V.; Cossi, M.; Cammi, R.; Mennucci, B.; Pomelli, C.; Adamo, C.; Clifford, S.; Ochterski, J.; Petersson, G. A.; Ayala, P. Y.; Cui, Q.; Morokuma, K.; Malick, D. K.; Rabuck, A. D.; Raghavachari, K.; Foresman, J. B.; Cioslowski, J.; Ortiz, J. V.; Stefanov, B. B.; Liu, G.; Liashenko, A.; Piskorz, P.; Komaromi, I.; Gomperts, R.; Martin, R. L.; Fox, D. J.; Keith, T.; Al-Laham, M. A.; Peng, C. Y.; Nanayakkara, A.; Gonzalez, C.; Challacombe, M.; Gill, P. M. W.; Johnson, B.; Chen, W.; Wong, M. W.; Andres, J. L.; Gonzalez, C.; Head-Gordon, M.; Replogle, E. S.; Pople, J. A. Gaussian03; Gaussian, Inc.: Pittsburgh, PA, 2003

(11) Goddard, R.; Heinemann, O.; Krüger, C. Acta Crystallogr. 1997, C53, 1846 .

(12) Meot-Ner, M. Chem. Rev. 2005, 105, 213. 